\title{
A Guinier Camera for SR Powder Diffraction: High Resolution and High Throughput
}

\author{
D. Peter Siddons ${ }^{\mathrm{a}}$, Steven L. Hulbert ${ }^{\mathrm{a}}$, Peter W. Stephens ${ }^{\mathrm{b}}$

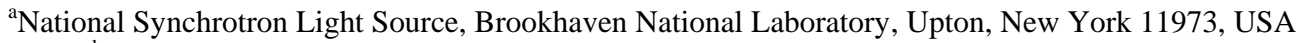 \\ ${ }^{\mathrm{b}}$ University of New York at Stony Brook, Stony Brook, New York 11794, USA
}

\author{
National Synchrotron Light Source \\ Brookhaven National Laboratory \\ P.O. Box 5000 \\ Upton, NY 11973 \\ www.bnl.gov
}

Notice: This manuscript has been authored by employees of Brookhaven Science Associates, LLC under Contract No. DE-AC02-98CH10886 with the U.S. Department of Energy. The publisher by accepting the manuscript for publication acknowledges that the United States Government retains a non-exclusive, paid-up, irrevocable, worldwide license to publish or reproduce the published form of this manuscript, or allow others to do so, for United States Government purposes. 


\section{DISCLAIMER}

This report was prepared as an account of work sponsored by an agency of the United States Government. Neither the United States Government nor any agency thereof, nor any of their employees, nor any of their contractors, subcontractors or their employees, makes any warranty, express or implied, or assumes any legal liability or responsibility for the accuracy, completeness, or any third party's use or the results of such use of any information, apparatus, product, or process disclosed, or represents that its use would not infringe privately owned rights. Reference herein to any specific commercial product, process, or service by trade name, trademark, manufacturer, or otherwise, does not necessary constitute or imply its endorsement, recommendation, or favoring by the United States Government or any agency thereof or its contractors or subcontractors. The views and opinions of authors expresses herein do not necessarily state to reflect those of the United States Government or any agency thereof. 


\title{
A Guinier Camera for SR Powder Diffraction: High Resolution and High Throughput.
}

\author{
D. Peter Siddons ${ }^{\mathrm{a}}$, Steven L. Hulbert ${ }^{\mathrm{a}}$, Peter W. Stephens ${ }^{\mathrm{b}}$

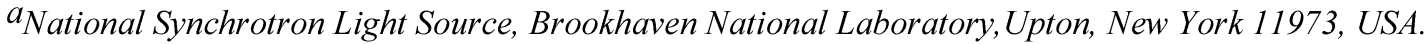

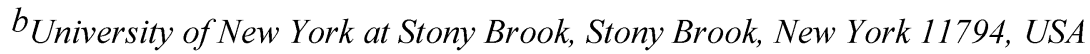

\begin{abstract}
The paper describe a new powder diffraction instrument for synchrotron radiation sources which combines the high throughput of a position-sensitive detector system with the high resolution normally only provided by a crystal analyser. It uses the Guinier geometry [1] which is traditionally used with an x-ray tube source. This geometry adapts well to the synchrotron source, provided proper beam conditioning is applied. The high brightness of the SR source allows a high resolution to be achieved. When combined with a photon-counting silicon microstrip detector array, the system becomes a powerful instrument for radiation-sensitive samples or time-dependent phase transition studies.
\end{abstract}

Keywords: Powder diffraction, High Resolution.

PACS: $07.85 . Q \mathrm{e}$

\section{GUINIER GEOMETRY}

The Guinier geometry [1] has been used with laboratory sources for many years. It is capable of providing highresolution powder patterns from large samples using low-brightness sources such as a standard x-ray tube. Figure 1 shows the geometry. It can take two forms, one (a) optimized for low diffraction angles, and the second (b) for high angles. A production instrument would allow rapid transition between these two modes, requiring primarily a simple translation of the circle along the incident beam, and provision of a second sample holder.

\section{OPTICS FOR SYNCHROTRON USE}

Synchrotron radiation beams are typically highly collimated or slightly convergent, depending on the intended setup at the beamline. Powder diffraction instruments are installed at both types of beamline, usually in some form of the Debye-Scherrer geometry, with the sample at the center of the diffractometer and the detector system rotating about that point. In parallel-beam instruments the detector is usually a combination of an analyzer crystal and a point detector. In modern designs this single detector may be augmented by a set of several identical systems mounted on the detector arm and rotating together to increase the throughput of the instrument. Beamlines with focusing optics typically use a position-sensitive detector and generally achieve lower resolution than the analyzer instruments, but much higher throughput.

The instrument described here tries to provide both high resolution and high throughput. It is a strongly focusing device, identical to the classical Guinier geometry, but scaled up to allow angular resolutions at the $10^{\wedge}-4$ level or better. Its intrinsic resolution is determined by the size of the focal spot at the Rowland circle and the detector spatial resolution. 
(a)

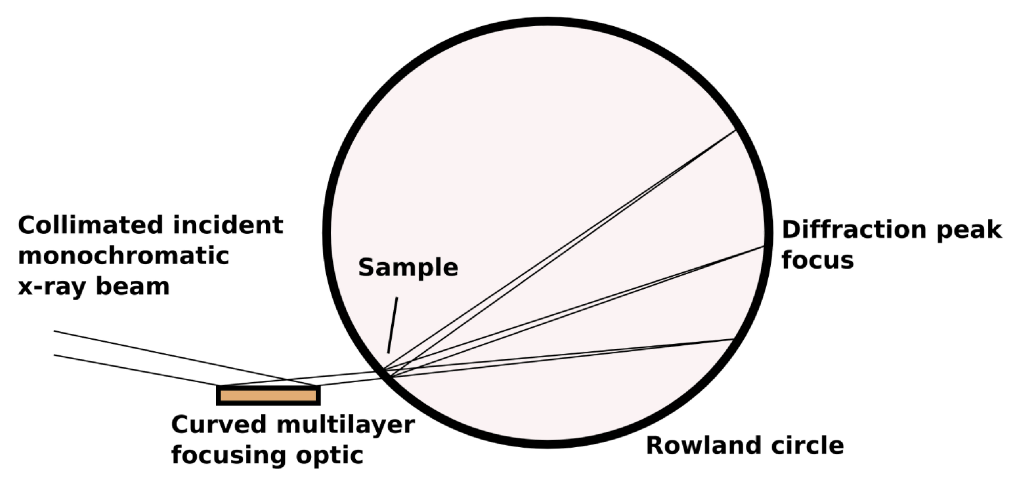

(b)

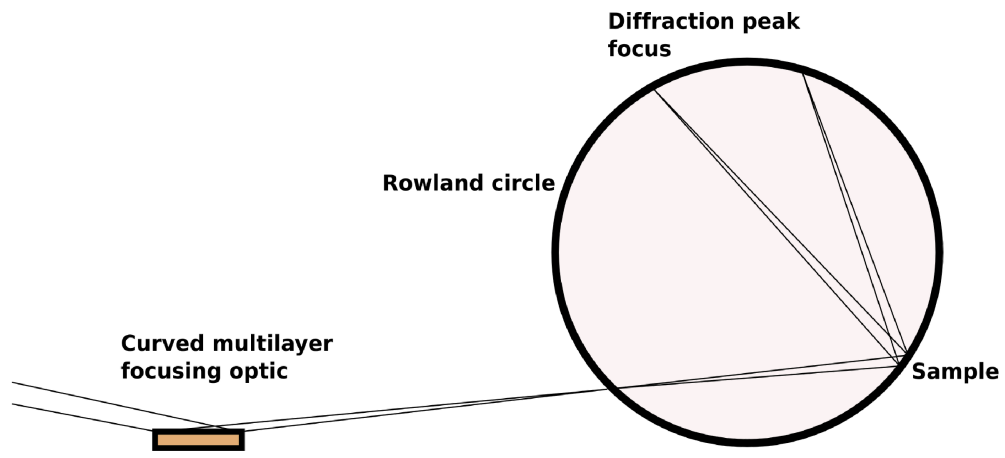

FIGURE 1. The Guinier geometry is a Rowland circle construction which can take two forms. (a) is optimal for low Bragg angle peaks and operates in transmission. (b) is optimized for high Bragg angle peaks and operates in reflection.

\section{THE SILICON STRIP DETECTOR}

The advantage of this geometry is only properly realized with an efficient, low-noise, high-resolution position sensitive detector. We have developed such a detector based on BNL's HERMES integrated circuit [2] and silicon microstrip arrays produced at BNL. It consists of a monolithic array of silicon diodes coupled to a set of applicationspecific integrated circuits designed at BNL. This allows each diode to detect $\mathrm{x}$-rays with good energy resolution and hence low noise. The sensitive thickness of the diodes is $0.4 \mathrm{~mm}$, allowing efficient detection up to about $12 \mathrm{keV}$. The array used for the tests reported here had 384 strips each $0.125 \times 4 \mathrm{~mm}^{\wedge} 2$, read out by 12 chips. Each chip has 32 full counting channels. Each channel consists of charge-sensitive preamplifier, shaping amplifier and a set of three discriminators and 24-bit counters. A choice of four shaping time constants is provided, from 0.5 to 4 microseconds. Two gain setting are provided, one having a maximum photon energy of $20 \mathrm{keV}$, and one with $40 \mathrm{keV}$ maximum. Since the photon energy used for these experiments was $8 \mathrm{keV}$, we used the high gain setting. Also, the detector was operated near room temperature and under these conditions the optimum performance is achieved with the shortest shaping time of 0.5 microseconds. It is capable of operating down to below -20 degrees Celsius if required for energy resolution reasons. This was not necessary for these experiments. It became clear from these experiments that there would be some advantage in having a wider detector. The line shapes measured show minimal asymmetry. Since the effects of asymmetry on the lineshape are well-known it would probably be of benefit to use a sufficiently wide detector such that some asymmetry is observed in order to increase the intensity detected. A factor of 2 or three would probably not be unreasonable.

\section{FOCUSING OPTIC}

If high resolution is to be achieved, we need an optical system which provides a good energy resolution, and at the same time provides a strongly demagnified image of the source at the detector. We would like the beam size at the sample to be large, to ensure as many powder particles are intercepted by the beam as possible, and we need a small focal spot (or line) to provide the required good Bragg angle resolution. While asymmetric crystal 


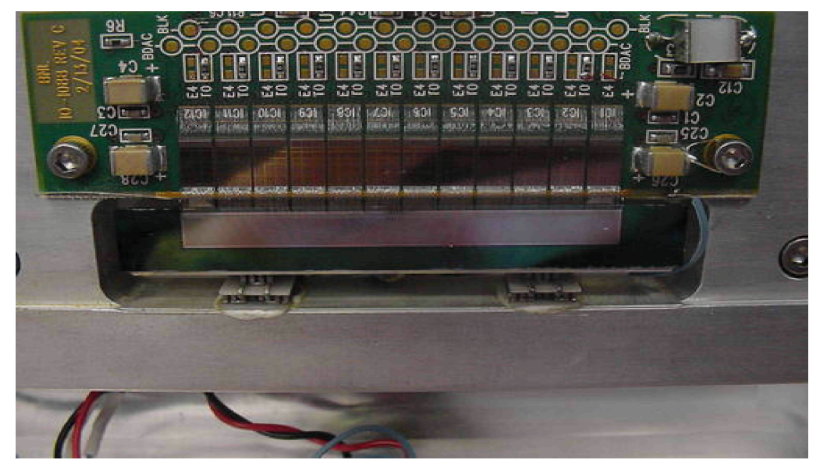

FIGURE 2. The silicon diode microstrip array. The aluminum frame is water-cooled to prevent overheating of the readout chips, and to provide a sink for the heat produced by the Peltier coolers when the detector is operated below ambient temperature.

monochromators can provide reasonable energy resolution and a small focus, they cannot provide a large beam cross-section at the sample in a synchrotron context because of the large asymmetry necessary to maintain the Cauchois geometry and hence good energy resolution. We chose to use the standard beamline 2-crystal monochromator to provide the high energy resolution, and to use a secondary optic to provide the strong focusing. We need to take the collimated beam from the beamline monochromator $(1.5 \mathrm{~mm} \mathrm{x} 4 \mathrm{~mm})$ and focus it down to as small a line focus as possible. The ultimate resolution of this instrument depends on the size of this focus and the detector spatial resolution. To perform this focusing, the beam downstream of the focusing optic will have a convergence of about $2 \mathrm{mr}$. This implies a range of incidence angles on the optic of $1 \mathrm{mr}$. A grazing-incidence mirror could do the job, since typical grazing angles are $3 \mathrm{mr}$ or more (depending on the mirror coating). Assuming an average grazing angle of $2 \mathrm{mr}$, the mirror would need to be almost $1 \mathrm{~m}$ long. This was not practical for us, since the instrument would then not fit in the available space. We chose to use a multilayer mirror to increase the grazing angle and maintain a high reflectivity. The multilayer used was a $\mathrm{W} / \mathrm{SiC}$ multilayer grown on a standard silicon device wafer, and had a d-spacing of around $4 \mathrm{~nm}$, providing a grazing angle of 1 degree at $8 \mathrm{keV}$. It was mounted in a simple spring-loaded bender, illustrated below, and bent to the correct radius by inspection of the focal spot using a YAG scintillator viewed by a video camera.

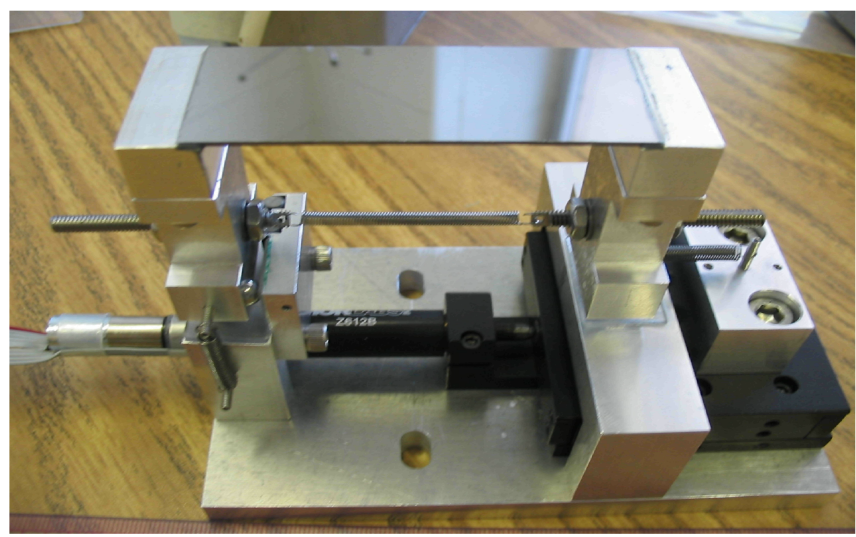

FIGURE 3. The multilayer mirror mounted on its bender. The bender is a simple ' $U$ ' bender, actuated by stretching a tension spring using a motorized translation stage. The feet of the ' $U$ ' ride on steel balls against a glass plate, minimizing any stiction. The mount is kinematic to minimize any undesired twist.

Test data for the system was collected using Maltose as a test material. It makes a good test, since it has many lines, even at low angles, and its structure is well-known. The system was operated at $8 \mathrm{keV}$, primarily because the available bendable multilayer could accept a significant beam aperture at that energy. Unlike a crystal analyzer, the momentum resolution of the instrument is energy-dependent since it offers a constant angular resolution, whereas a crystal analyzer offers constant momentum resolution. The data was taken using the $48 \mathrm{~mm}$ long array illustrated above, and stepping through the required angular range in 1.5 degree steps (the detector covered 2.6 degrees). Obviously a full instrument would have a complete position-sensitive detector covering the entire range. Below we 
show the data, together with a Reitveld fit using a Voigt line profile. We also show a crystal analyser scan of the same material for comparison. The crystal scan was collected using $17 \mathrm{keV}$ radiation and so it's angle scale is roughly half that for the Guinier data. It consisted of 3274 points of 1 second each, i.e. about 60 minutes for the scan. The Guinier instrument covered a similar d-spacing range in 14 exposures of 50 seconds each, collecting roughly 5 times the counts. If we had the full detector array, this would represent a speed increase of 300 for equivalent counting statistics, a worthwhile improvement for many applications. If that detector had 3 times wider diodes (a practical possibility), then the speed gain would approach 1000. The ideal instrument would have an improved multilayer optic, with improved figure error and coating quality, smaller d-spacing and would be much longer to allow operation at higher energies without losing beam aperture. It would have a full detector array of about 7000 elements covering 90 degrees of the Rowland circle (45 degrees in 2-theta). The detector would be mounted in such a way that it could switch between the two modes of operation (as shown in fig. 1) quickly and easily. Such an instrument would be a powerful tool for chemistry and materials science.

(a)

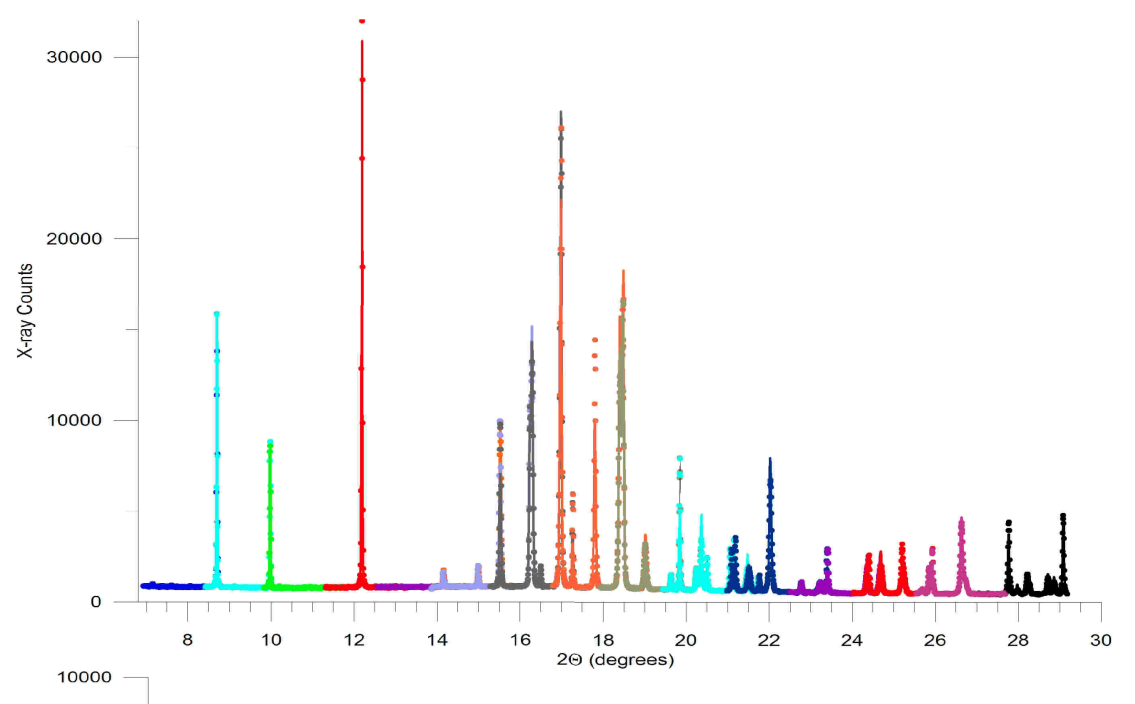

(b)

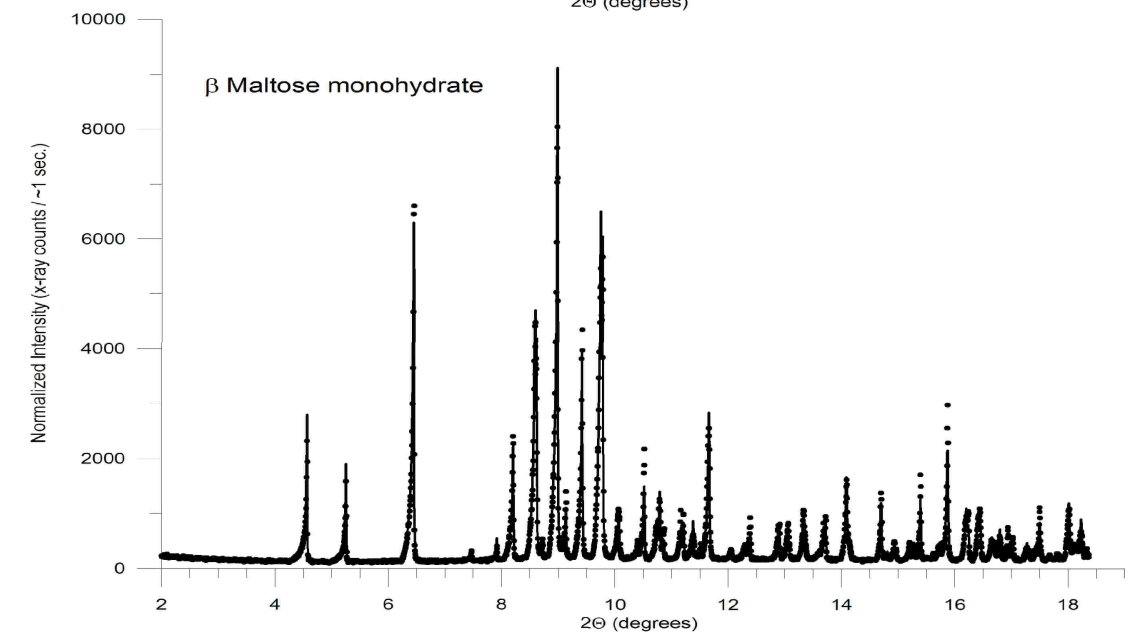

FIGURE 4. Data collected using (a) the Guinier camera and (b) a crystal anayzer instrument.

\section{REFERENCES}

1. A. Guinier, 'X-ray Crystallographic Technology', Hilger \& Watts, London, 1952.

2. G. De Geronimo et al, IEEE Trans. Nucl. Sci, 50 (2003) 885-891 